Université du Québec à Chicoutimi (Canada) Claudia Gagnon Université de Sherbrooke (Canada) Johanna Bisson

Lennoxville Vocational Training Center (Canada) Andréanne Gagné

Université de Sherbrooke (Canada) Stéphane Dupuis

Centre de services scolaire des Sommets (Canada) Manon Larouche

Centre de formation professionnelle Alma (Canada) Marie Alexandre

Université du Québec à Rimouski (Canada) Chantale Beaucher

Université de Sherbrooke (Canada)

\section{Transformations des pratiques enseignantes en formation professionnelle au Québec avec l'arrivée de la COVID-19}

Changes in teaching practices across Québec's professional training centres due to COVID-19

doi: $10.18162 /$ fp.2020.682

\section{ésumé}

Les mesures sociosanitaires exigées par le gouvernement du Québec pendant le contexte de pandémie ont affecté de manière importante les pratiques enseignantes dans tous les centres de formation professionnelle. Des transformations liées aux contenus des programmes, des processus de formation et des éléments organisationnels ont été réalisées par les équipes enseignantes pour assurer une formation de qualité à la future maind'œurre québécoise. Ancrés dans le vécu d'acteurs du milieu, les changements mis en place soulèvent des enjeux importants quant aux exigences de qualification, au développement de la compétence numérique et à leur pérennité dans le temps.

\section{Mots-clés}

Formation professionnelle, pratiques enseignantes, changement

Abstract

The social and sanitary measures imposed by the Québec government during the pandemic have substantially impacted teaching practices throughout professional training centres. Teams of teachers have transformed program content, training processes, and organizational elements to ensure that Québec's upcoming labor force is professionally qualified. Based on the experiences of actors in the field, these changes raise important issues concerning the requirements for qualification and the acquisition and continuous updating of digital skills..

Keywords Professional training, teaching practices, change
Dans le contexte de pandémie, plusieurs métiers essentiels au fonctionnement de la société québécoise qui sont enseignés dans les centres de formation professionnelle (CFP), tels que ceux en santé, en mécanique, en soutien informatique, en transport routier, en comptabilité et en horticulture, ont occupé une place de premier plan tant leur rôle devenait incontournable au quotidien. Le moment semble tout désigné de faire le point sur les défis relevés au secteur de la formation professionnelle (FP), dans le contexte où l'enseignement doit s'effectuer principalement à distance et qu'en laboratoires ou en ateliers, le gouvernement exige un maximum de 15 élèves.

\section{Le contexte de la FP au Québec : défis et tensions}

Au Québec, la FP qualifie chaque année plus de 120000 élèves âgés de 16 ans et plus dans 21 secteurs économiques ${ }^{1}$ (Banque de données des statistiques officielles sur le Québec [BDSO], 2019). Jouant un rôle économique central (Masdonati, Fournier et Pineault, 2015), à la jonction des équipes des $\mathrm{CFP}$, des centres de services scolaires (CSS), des universités et des milieux du travail, elle possède un positionnement unique créant diverses tensions entre une formation générale pour exercer un métier et les exigences de qualifications, entre les besoins de main-d'œuvre et la qualité de la formation, entre l'hétérogénéité des élèves et les particularités des personnes enseignantes, ainsi qu'entre des pratiques enseignantes déjà près de la formation hybride et d'autres ne pouvant s'effectuer à distance, en raison de la nécessaire formation pratique. Ce texte montre des défis quotidiens de la FP accentués par le contexte de pandémie, puis brosse un portrait des répercussions concrètes sur les pratiques enseignantes du confinement et des mesures sociosanitaires dans différents programmes de FP. 


\section{Défi 1 : qualifier en contexte réel ou le plus authentique possible}

Les programmes de FP se découpent en compétences évaluées individuellement. En temps normal, la planification de la formation repose sur la détermination de la séquence d'acquisition des compétences de métier (Tremblay, 2016) et, pour chaque compétence, sur l'identification des modalités de formation appropriées au développement des savoirs professionnels (connaissances, habiletés, attitudes, perceptions et stratégies) et à l'exercice de la compétence (Gagnon et Tremblay, 2016a), en déterminant la durée et l'ordre d'enseignement, en choisissant les stratégies et les moyens d'enseignement (Gagnon et Tremblay, 2016b). Certes, le développement d'une compétence n'est jamais achevé, mais en FP, la réussite de chaque compétence s'avère essentielle à la qualification des individus correspondant au seuil d'entrée sur le marché du travail (Ministère de l'Éducation, du Loisir et du Sport, 2005). Le processus de développement d'une compétence demande toutefois du temps pour l'acquisition de connaissances, l'entraînement nécessaire et la réalisation de la tâche dans des situations variées (Ibid.).

Ainsi, pour qualifier la main-d'œuvre québécoise, la FP doit se dérouler en contexte réel, en tentant « de reproduire, le plus fidèlement possible, la "vraie vie " professionnelle avec ses outils, son matériel, son équipement» (Faucher et Roy, 2016, p. 77). Outre la classe, la formation s'offre dans des environnements variés : atelier, garage, serre, chantier, laboratoire, salle à manger, etc. Elle s'y déroule selon des modes d'enseignement diversifiés (traditionnel, enseignement individualisé ${ }^{2}$, en présentiel et à distance) et comporte des modalités d'organisation qui relèvent d'un partenariat important avec le monde du travail (stages, alternance travail-études, apprentissage accru en milieu de travail) (Gagnon, 2020). Afin de répondre aux normes sociosanitaires prescrites par la santé publique dans le contexte de la pandémie, la planification de l'enseignement et les pratiques qu'elle sous-tend ont été revues.

\section{Défi 2 : L'hétérogénéité des élèves}

La FP s'adresse par ailleurs à un effectif hautement hétérogène quant à l'âge, la motivation, le parcours scolaire, l'expérience personnelle et professionnelle (Coulombe, Doucet, Maltais, De Champlain, et Bizot, 2019 ; Gagnon et Beaucher, 2016 ; Mazalon et Bourdon, 2015). Près de $60 \%$ des élèves sont considérés comme étant vulnérables ou présentant des besoins particuliers, dont des troubles d'apprentissage, de comportements ou de santé mentale (Beaucher, Coulombe, Murphy, Gagnon, Doucet et Maltais, 2020). Au quotidien, cette situation comporte des défis liés à une mobilisation des acteurs des CFP (enseignants, professionnels, directions, etc.) pour accompagner tous les élèves dans leurs apprentissages et revisiter les pratiques pédagogiques dans une logique d'éducation accessible et non discriminatoire pour tous (Breton, 2020; Philion, Bourrassa, Lanaris et Pautel, 2016). En contexte de pandémie, ce défi soulève des enjeux encore plus prégnants, parmi lesquels l'anxiété générée chez certains élèves, aux difficultés financières et à celles de conciliation études-travail-famille exacerbées chez des parents poursuivant des études en FP.

\section{Des défis spécifiques liés au personnel enseignant}

Pour relever ces précédents défis, la FP doit compter sur un personnel enseignant bien formé, et là encore, des défis se posent. Embauchées pour leur expertise et leur expérience de métier, les personnes enseignantes sont âgées en moyenne de 42 ans et amorcent le baccalauréat en enseignement à temps

2 - Formation et profession 28(4 hors-série), 2020 
partiel une fois en emploi dans un CFP. Pour plusieurs, il s'agit d'un virage extrême entre le métier exercé et l'enseignement de ce métier (Balleux, 2011). De surcroît, cette formation étalée sur une période d'environ 10 ans se juxtapose à des conditions d'embauche précaires. En 2015-2016, 9979 personnes enseignaient en FP au Québec (BDSO, 2018), dont 27 \% étaient permanentes, 33 \% non permanentes et $40 \%$ en appoint (à taux horaire).

\section{Nécessaire adaptation et changement des pratiques enseignantes}

Les mesures de distanciation sociale, d'hygiène et de salubrité exigées par le gouvernement du Québec dans tous les CFP ont affecté de manière importante les routines et les pratiques de la FP. Les enjeux sont majeurs puisqu'ils retardent l'entrée sur le marché du travail de milliers d'élèves, placent le personnel enseignant en situation encore plus précaire et mettent à l'épreuve sa capacité d'adaptation et de résilience. Quels changements ou quels ajustements ont été apportés aux pratiques enseignantes pour qualifier aux métiers de la FP?

\section{Pratiques enseignantes et transformations}

Les pratiques enseignantes en FP constituent un système complexe d'actes singuliers de la part de l'enseignant et de significations qu'il leur accorde (Altet, 2002; Gagnon, 2007; Marcel, 2004). Elles comprennent les pratiques administratives et collaboratives, de supervision et liées à l'accompagnement des élèves en stage, de tutorat et d'enseignement (Beaucher et Gagnon, 2016). Effectuées à l'intérieur ou hors du CFP, ces dernières comportent trois phases : planification, intervention auprès des élèves et évaluation des compétences. Bru (1992) a défini trois catégories de variables d'action sur lesquelles l'enseignant adopte différentes façons de faire. Les objets réfèrent aux savoirs enseignés ou utilisés (tâches, thèmes, sujets). Le processus renvoie au déroulement des activités, à la dynamique de l'apprentissage, à la répartition des initiatives, etc. Enfin, les éléments organisationnels concernent les lieux et l'organisation de l'espace où se déroule la formation, les moyens utilisés (matériel didactique, équipements, outils), les personnes impliquées, ainsi que le temps de formation (durée, moment) (Bru, 1992).

Les transformations de pratiques sont définies, pour leur part, par des ajustements, des adaptations et des changements (Dionne, 2007). Les adaptations émergent de la nécessité d'améliorer une situation ou de résoudre des problèmes liés à l'action; elles font suite à une réflexion dans et sur l'action (Couture, Dionne, Savoie-Zajc et Aurousseau, 2012 ; Schön, 1994). Ainsi, les événements soudains liés au contexte de pandémie ont déstabilisé les routines établies par les équipes enseignantes et les organisations scolaires et professionnelles, se soldant ainsi en ajustements divers. Ces ajustements, circonstanciels et temporaires, mèneront possiblement à des changements de pratiques qui, eux, représentent des modifications pérennes, réfléchies, appuyées et légitimées (Guertin, 2012 ; Lenoir, 2012). 


\section{Des cas analysés sur la base de récits de pratique}

Trois enseignants (E1, E2, E3), une personne responsable du service aux entreprises (P1) et deux conseillers pédagogiques ( $\mathrm{CP} 1$ et $\mathrm{CP} 2)$ ont accepté de partager l'expérience vécue dans leurs $\mathrm{CFP}$ entre mars et juin 2020. Suivant une démarche ancrée dans le vécu de ces personnes, près de l'étude de cas proposée par Yin (1994) et basée sur les récits de pratique (Desgagné, 2005), des « cas-récits » ont été réalisés à partir de réponses écrites et orales obtenues à des questions portant sur les transformations des pratiques enseignantes en contexte de pandémie (particularités du programme, modes d'organisation, enseignement-apprentissage, contraintes, etc.).

Les « cas-récits » concernent les programmes suivants : 1) Mécanique industrielle de construction et d'entretien (MICE) (DEP de 1800 heures du CFP Windsor, 2) Secrétariat (DEP de 1485 heures des CFP Windsor, Magog et Asbestos, 3) Soutien informatique (DEP de 1800 heures du CFP Alma, 4) Horticulture et jardinerie (DEP de 1335 heures du CRIFA de Coaticook) et, dans le secteur de la santé, 5) Assistance à la personne en établissement et à domicile (DEP de 870 heures), Assistance à la personne en résidences privées pour aînés (AEP de 180 heures) et Assistance à la personne en centre hospitalier de soins de longue durée (CHSLD) (AEP de 375 heures $^{3}$ du Lennoxville Vocational Training Center (LVTC).

L'analyse de contenu (Mayer et Deslauriers, 2000; Paillé et Mucchielli, 2016) a permis de dégager des éléments récurrents ou des convergences entre les cas, tout en mettant en lumière leurs particularités (Yin, 1994).

\section{Transformations de pratiques : objets, processus et éléments organisationnels}

Les cas-récits décrivent les situations et les problèmes en donnant accès aux enjeux et aux choix réalisés pour ajuster ou changer les pratiques enseignantes aux plans de l'objet, du processus et des éléments organisationnels pendant le confinement et au moment de la réouverture des CFP.

\section{Des transformations qui concernent peu les objets de formation}

Tout d'abord, le contenu de formation s'est limité, pour l'ensemble des cas, aux éléments essentiels prévus dans les programmes d'études. Contrairement à ce qui se fait généralement, le personnel enseignant n'a pratiquement pas fourni de contenu d'enrichissement aux élèves. Quoi qu'il en soit, «si les formations ont été offertes, c'est parce que la pleine acquisition des compétences était possible, donc autant la pratique que la théorie» (CP1). Néanmoins, l'ordonnancement des contenus a généralement été modifié et renvoie au processus de formation.

Seuls les contenus de formation des préposés aux bénéficiaires $(\mathrm{PAB})$ dans le secteur de santé ont été fortement remis en question, avec le manque criant de personnel qualifié pour soigner nos aînés en CHSLD. Le gouvernement a alors exigé des CFP qu'ils forment des préposés en trois mois seulement. Pour bien comprendre, il faut savoir qu'un DEP de $\mathrm{PAB}$ " régulier » de 870 heures et qu'une AEP de 180 heures pour former des PAB en résidences privées existaient déjà, bien avant cette demande gouvernementale. Des formations sur mesure sont également offertes en collaboration avec les centres

4 - Formation et profession 28(4 hors-série), 2020 
intégrés universitaires de santé et de services sociaux (CIUSSS) pour permettre aux élèves en santé de travailler à titre de préposés pendant leurs études. Cependant, les CFP ont dû créer une formation accélérée de 375 heures permettant d'accéder à une qualification minimale d'assistance à la personne en CHSLD. Ils ont ciblé les compétences essentielles, voire même des parties de compétences des programmes existants pour aider les personnes en perte d'autonomie, celles atteintes de maladies dégénératives ou de problèmes de santé mentale. Les personnes qualifiées pour cette AEP travailleront uniquement en $\mathrm{CHSLD}^{4}$.

\section{Des transformations qui bouleversent le processus de formation}

Les cas-récits partagés montrent des réorganisations liées au déroulement des activités en classe ou en stage pour convenir à la formation à distance. Pour certains enseignants, cette période de réorganisation des contenus a été particulièrement intense :

[...] moi, je n'y croyais pas, parce que je me voyais mal expliquer des choses pratiques à distance. [...] La phase ultime de gros stress a été la planification des cours. J'ai compté le nombre d'heures qu'il me restait à donner aux élèves. Il fallait que je récupère trois semaines de cours. On nous a demandé pour chaque compétence combien d'heures on estimait avoir besoin de formation en ligne et combien d'heures en présentiel (E1).

Si poursuivre l'apprentissage des contenus «théoriques » à distance était possible, le processus de développement des compétences était rapidement limité. Ainsi, pour la majorité des programmes, la partie « théorique » des compétences a été enseignée pendant le confinement. La partie « pratique »et l'évaluation ont été réalisées lors du déconfinement, avec un écart de temps important. En horticulture, normalement, la formation est donnée en classe, en atelier, en serre et sur des terrains du centre et en dehors de celui-ci. Chaque élève a la responsabilité de semis, de bouturage et d'entretien de différents végétaux; l'approche par projet est très présente et le programme comprend 300 heures de stages en ATE. Au mois d'avril, la théorie est abordée le matin et l'après-midi, les élèves sortent dehors ou vont dans la serre pour pratiquer. Ce processus de formation a été revu :

J'ai revu toute ma théorie pendant le confinement, et j'ai trouvé des éléments pratiques ou préru du visuel pour leur montrer l'aspect pratique, ce que je ne fais pas habituellement parce que je sors dehors. Juste positionner mon échelle de façon sécuritaire dans l'arbre - généralement je passe et je leur montre comment faire et je reprends ceux qui ne le font pas correctement. Heureusement, j'avais pris des photos d'élèves des dernières années, ça m'a aidé à leur montrer (E1).

Dans le cas des programmes de Secrétariat, l'impact s'est moins fait ressentir. Le programme est enseigné par quatre équipes enseignantes réparties dans trois CFP selon trois modèles organisationnels différents en enseignement individualisé. Deux équipes offrent une formation en classe, une équipe offre un enseignement hybride (en classe et à distance) en mode synchrone et une équipe offre une formation totalement à distance, en mode asynchrone. Cette dernière a aidé les autres équipes à migrer vers la formation à distance (FAD). Pour toutes les équipes, les élèves étaient pour la plupart autonomes en FAD : 
En Secrétariat [...], nous avons surtout retenu l'évaluation de compétences pendant quelques semaines, même si les apprentissages étaient terminés. Les élèves ont géré [...] leur progression dans les quelques compétences ouvertes. Plusieurs ont conservé des devoirs ou des activités de synthèse pour se préparer à l'examen, une fois l'accès aux salles de test permis (CP1).

En santé et en soutien informatique, les équipes se sont rapidement réorganisées et ont donné les cours à distance. Du soutien informatique est normalement offert à l'aide d'une plateforme virtuelle suivant une approche programme, en présentiel et à distance, si désiré par les élèves, pour certaines compétences. 240 heures en ATE (stages terminaux) sont également prévues au programme. E3 soutient que, dès le début du confinement, un sondage a été envoyé aux élèves pour évaluer l'intérêt à poursuivre la formation en ligne : "Toute la théorie devait être donnée en ligne. Le contexte d'apprentissage devait être favorable. [...] Les vingt élèves de première année étaient intéressés » (E3).

En plus de chambouler le processus d'acquisition d'une compétence, la situation liée à la COVID-19 a suscité d'autres transformations dans le déroulement des activités lors du retour dans les CFP. En horticulture, E1 indique, à propos des démonstrations : "Je devais le faire deux fois pour respecter la distanciation: une moitié du groupe devant moi pendant que je faisais travailler l'autre moitié ailleurs, et plus tard avec l'autre groupe ».

Des réaménagements ont également été apportés aux stages et aux programmes offerts en alternance travail-études (ATE). C'est le cas en MICE, où les 120 heures prévues en ATE ont été réalisées dans les ateliers du $\mathrm{CFP}^{5}$, exigeant des enseignants qu'ils prennent en charge la formation de cette période pour les élèves présents. Ils ont revu le calendrier de formation et les horaires d'enseignement. Toutes les activités de supervision de stage ont été annulées. "À la rentrée, à moins d’un avis général, les entreprises devront être recontactées, afin d'analyser la possibilité de réaliser ou non des stages dans leurs murs » (CP1). De même, dans le secteur de la santé où une grande partie de la formation se déroule en stage, "plusieurs stagiaires ont été refusés dans les établissements de santé pour diminuer les risques de contagions, particulièrement pour les compétences en maternité et en chirurgie» (CP2). En horticulture, deux des trois séquences de cinq jours de stages en ATE prévues ont été annulées, alors que les élèves ont réalisé le stage dans les centres jardins, ceux-ci faisant partie des services essentiels pendant la pandémie. Or, certains centres jardins ne voulaient pas accueillir de stagiaire dans ce contexte, donc le stage a duré seulement trois jours, comprenant le samedi et le congé férié du mois de mai, ce qui ne se fait pas dans le calendrier scolaire habituel.

Enfin, en ce qui a trait à l'évaluation des compétences développées à distance, celle-ci ne semble pas a priori convenir à la FP, car elle s'effectue normalement sur la base de gestes professionnels maîtrisés et observés dans des situations près de la réalité du marché du travail. En santé, « seules les techniques (ateliers et laboratoires) et les examens étaient vus ou faits au CFP avec demi-groupe respectant la distanciation » (CP2). En soutien informatique, l'évaluation individuelle a été réalisée en ligne en synchrone pour trois compétences qui étaient en cours au moment du confinement. "Les deux autres étant pratiques ont été reportées à l'an prochain» (E3). 


\section{Des transformations qui concernent surtout les éléments organisationnels}

En ce qui a trait aux éléments organisationnels, l'espace où se déroule la formation, les moyens utilisés (matériel didactique, équipements, outils), les personnes impliquées et le temps de formation (durée, moment) ont aussi subi des transformations importantes pour répondre aux nouvelles normes sociosanitaires.

\section{Transformation des lieux : l'impossible accès au contexte réel}

Concernant les lieux de formation (classe, ateliers, laboratoires, etc.), la principale difficulté, pendant le confinement, a été la fermeture totale puis l'ouverture partielle des CFP qui a contraint des équipes enseignantes à séloigner des environnements reproduisant le milieu de travail et à mettre sur pause tous les stages en entreprise. De la classe et du contexte authentique de formation, les enseignants ont dû dans un premier temps basculer vers la classe virtuelle pour les contenus théoriques.

Dans un deuxième temps, au moment de la réouverture des CFP, des réaménagements ont été nécessaires dans les classes et les ateliers : postes de désinfection, bureaux disposés à deux mètres de distance, sens uniques pour indiquer la circulation dans les classes et les bâtiments. En horticulture, réorganiser l'espace pour accueillir les élèves en classe a demandé quelques jours de préparation, mais a été facilité parce qu'il restait essentiellement de la formation pratique à faire à l'extérieur :

J'ai regardé dans le jardin pédagogique etj'ai évaluéles besoins en entretien des jardins. J'ai séparé en parcelles pour que chaque élève ait sa parcelle pour respecter la distanciation sociale. J'ai aussi planifié pour les déplacements sur les tâches en dehors du centre mais, dans ce cas, tout le monde portait des masques (E1).

Dans les CFP, des lieux qui ne concernent généralement pas l'enseignement ont aussi été transformés. C'est le cas notamment des cafétérias, des salles de dîner et des salles de toilettes, pour limiter les déplacements, disposer de plus d'espace pour offrir l'enseignement et effectuer le nettoyage requis.

\section{Transformation des moyens : le virage technologique}

De nouveaux moyens pour communiquer et enseigner ont été adoptés, le matériel didactique utilisé normalement en classe ayant été transformé pour convenir à la formation à distance (FAD) et les pratiques technopédagogiques mises à jour pour l'ensemble des personnes enseignantes.

Avant la pandémie, certains programmes étaient offerts à distance, comme en secrétariat. D'autres utilisaient certains moyens technologiques sans toutefois dispenser la formation à distance et d'autres encore utilisaient très peu les technologies pour communiquer avec les élèves ou enseigner, comme c'était le cas en horticulture : "la CS voulait quion communique avec les élèves via leur adresse générée par la CS, mais le portail nétait pas utilisé avant le COVID-19, ni leur courriel - rarement de toute façon on faisait des communications par courriel aux élèves» (E1).

En outre, pour bien des enseignants, le matériel didactique utilisé dans le contexte régulier de formation a été modifié pour être adapté à la FAD :

[...] j'ai créé des dossiers par compétences [en ligne] (word, ppt, etc.). [...] J'ai revu chacun des documents pour m'assurer que tout était complet, parce que quand je le donne en classe, j’explique en même temps pour m'assurer qu'il n'y a pas de confusion (E1). 
Dans tous les CFP, du matériel informatique a été prêté au personnel enseignant et aux élèves afin d'enseigner ou d'apprendre à distance. Il a aussi été essentiel de former tout le personnel enseignant aux outils et aux principes pédagogiques de la FAD. Au LVTC, une équipe a été mise en place pour former et soutenir l'utilisation des technologies pour la FAD :

[Nous] avons organisé des ateliers avec un responsable RÉCIT pour initier les enseignants à Moodle. Nous avions deux groupes en parallèle avec des rencontres 1 ou 2 fois par semaine pour développer les compétences et créer les cours. [... Nous] avons rapidement grossi notre équipe avec un technicien, deux enseignants de la FGA, un enseignant de FP, puis deux conseillers RÉCIT selon les besoins. De mon côté, j'ai été sur du "sur mesure" pour mes profs en FP, [...] rencontres Zoom plusieurs fois/semaine individualisées, ateliers en ligne offerts 2 fois/semaine, ateliers et outils présentés rendus disponibles dans un groupe Google Drive (CP2).

En soutien informatique, ce qui a aidé, c'est que « la plateforme Moodle en était à sa deuxième année ce qui fait que les élèves ainsi que les enseignants la maîtrisaient déjà » (E3).

\section{Transformations pour des personnes impliquées : répercussions, collaborations et relation maître/ élèves.}

Le contexte de pandémie a eu des répercussions sur la tâche et le rôle des enseignants. En santé, dans le cas du nouveau programme de $\mathrm{PAB}$ :

l'elève a été jumelé avec un PAB par l'établissement de santé. Cet employé doit compléter des grilles d'observation afin d'assurer et de valider les apprentissages. L'enseignant, après avoir apprivoisé la FAD se voit devant un nouveau déf, car habitué de superviser directement 6 élèves, il est informé que pour ce nouveau programme une supervision indirecte de 22 élèves lui est confiée (CP2).

E3 souligne pour sa part que l'enseignant en soutien informatique : "doit offrir des capsules en ligne en direct avec l'élève lors de difficultés de compréhension de la matière. Il doit offrir du soutien technologique pour l'utilisation des différents outils de travail en ligne ». En parallèle, "l'élève doit être plus autonome et cela n'a pas été facile pour tous, car manque de motivation et de discipline» (E3).

Par ailleurs, une plus grande collaboration entre enseignants, professionnels et directions intra-CFP, interrégionale, voire même provinciale, a été nécessaire pour s'adapter au contexte de pandémie. C'est le cas du nouveau programme de formation en santé, mais également de toutes les équipes mobilisées pour réorganiser les espaces de façon à répondre aux normes sanitaires gouvernementales. En horticulture, E2 souligne :

j'ai travaillé davantage avec le magasinier et les concierges. Les concierges nous fournissaient les bouteilles de désinfectants et les guenilles. Avec le magasinier, je devais m'assurer qu'il y ait des outils prêts pour chaque élève (E2).

Pour les directions, plusieurs rencontres en ligne avec les CSS ainsi qu'avec le personnel du CFP ont été réalisées pour la coordination et la circulation rapide des informations. Ils ont prêté et remis du matériel aux milieux de santé (gants, masques, désinfectants, blouses), organisé des visites pour des dirigeants de la santé publique au cas où ils auraient besoin de lits et des espaces en santé. Ils ont coordonné des formations technopédagogiques, une distribution de matériel informatique donnant 
accès à Internet aux élèves qui en avaient besoin. Ils ont soutenu la préparation du retour en classe, développé un protocole pour s'assurer que les lieux soient sécuritaires et, parfois, géré des cas de COVID-19 déclarés.

Ce contexte unique a également eu des impacts sur la relation maître-élève et, conséquemment, sur la gestion de classe, la motivation des élèves et le temps pour rassurer les élèves anxieux :

Difficile la gestion de classe [en ligne]. On ne voit pas le non verbal. Habituellement [...] je vois les points d'interrogation dans leur visage, mais je ne voyais pas les élèves, [certains] faisaient autre chose en même temps, tout le monde intervenait à peu près n'importe quand. On a commencé à utiliser le chat, mais cétait trop de choses à gérer (E2).

\section{Discussion}

La qualification des élèves dans les CFP a été hautement ébranlée pendant la crise de la COVID-19. Le personnel enseignant s'est retrouvé, dans un premier temps, immobile, en attente de consignes gouvernementales, puis dans l'obligation d'offrir de la FAD selon ses capacités et suivant les mesures de distanciation sociale et d'hygiène pour le retour en CFP. Que l'on soit un enseignant expérimenté ou non, cette période a exigé nombreux ajustements, voire de nombreux changements de pratiques, tels que définis par Guertin (2012) et Lenoir (2012). Ces changements de pratiques soulèvent des enjeux importants quant aux exigences de qualification, au développement de la compétence numérique (Ministère de l'Éducation et de l'Enseignement supérieur [MEES], 2019), à leur pérennité dans le temps et au développement professionnel du personnel enseignant.

En effet, outre la formation dans divers contextes authentiques à l'intérieur des CFP, les stages en entreprise et la supervision de ceux-ci demeurent des modalités importantes pour favoriser la réussite des élèves et leur intégration sur le marché du travail (Savoie-Zajc et al., 2010). Quant à l'évaluation, bien que certaines mesures alternatives aient été explorées dans le contexte de la COVID-19, il est nécessaire de porter une attention aux évaluations en aide à l'apprentissage et la rétroaction effectuée par les enseignants (Roberge, 2017), particulièrement dans le contexte de la FAD (Plamondon et Ross, 2020). Quoi qu'il en soit, les évaluations aux fins de sanctions ne sont pas autorisées à distance; aussi est-il important, tant pour le ministère que pour les CFP, de réfléchir aux processus d'évaluation à distance (Leroux, 2017) et aux questions liées à la sécurité et aux modalités des épreuves aux fins de sanctions en FP.

Par ailleurs, le personnel enseignant, comme les élèves et d'autres acteurs du milieu, a rapidement développé la compétence numérique (MEES, 2019). Plusieurs équipes enseignantes neétaient pas préparées alors que d'autres fonctionnaient déjà en mode hybride ou totalement à distance (Alexandre, 2017, MEES, 2019). Les cas-récits montrent notamment le développement d'habiletés technologiques pour maîtriser les logiciels de la FAD, l'exploitation des outils technologiques pour l'enseignement, l'apprentissage, la collaboration et la production de contenu. Or, l'apport des technologies de l'information et de la communication (TIC) à la formation des élèves est largement tributaire de la manière de les utiliser et des contextes socioculturels (Alexandre, 2018; Collin et Karsenti, 2013; Plamondon et Ross, 2020). Comme cela a été montré en formation universitaire (Alexandre, 2019, Basque et Baillargeon, 2013), la planification et la réalisation d'un cours en FAD transforment les 
rôles de l'enseignant et posent des défis pour la relation enseignant-élève (Espinosa, 2020; St-Germain et Mazalon, 2020). Bien que la qualité soit actuellement l'un des principaux enjeux de la FAD et malgré le fait que l'usage des TIC en contexte éducatif ait été l'objet de nombreuses études, la pratique enseignante en FAD demeure à ce jour peu formalisée en FP. Force est de constater qu'en contexte "d'urgence ", les changements de pratiques à l'égard du numérique s'opèrent rapidement.

Dans le même sens, les ajustements réalisés soulèvent des questions quant à leur pérennité dans le temps et mettent en relief la notion de changement de pratiques lié au développement professionnel des enseignants (Marcel, 2005), lequel, suivant Uwamariya et Mukamurera (2005), est un processus de transformation par lequel les enseignants parviennent progressivement à améliorer, maîtriser et se sentir à l'aise dans leur pratique. Ce processus d'apprentissage accéléré vécu par des enseignants de la FP a permis d'améliorer les compétences professionnelles par la mobilisation des objets de formation et de ressources personnelles et environnementales de façon pertinente dans les situations de travail (Le Boterf, 2013). Si notre regard a porté sur les pratiques enseignantes de différents individus, l'évolution de celles-ci a été déterminée par les conditions matérielles, organisationnelles, relationnelles et culturelles particulières de travail dans la situation liée à la pandémie, d'où l'intérêt, a posteriori, d'aborder le développement professionnel des enseignants à travers la dimension collective, voire sociale ou sociétale (Gosselin, Viau-Guay et Bourassa 2014; Lefeuvre, Garcia et Namolovan, 2009; Wittorski, 2007).

\section{Conclusion}

Les transformations des pratiques enseignantes dans le contexte de pandémie sont marquées par trois temps : la fermeture complète des écoles (1), l'offre de formation à distance (2) et le retour en CFP des élèves (3). Au temps 1, l'annonce de la fermeture complète des CFP a déstabilisé toute la société incluant le monde scolaire. Par la suite, les équipes des CFP ont réorganisé les pratiques enseignantes (objets, processus, éléments organisationnels) en respectant le temps de formation et le nombre d'heures prescrites aux programmes. Le dénominateur commun aux changements de pratiques est certes d'assurer les exigences de qualifications aux différents métiers. Au temps 3, ces équipes semblent rester prudentes face à l'incertitude et au regain du virus parmi la population: "Tranquillement, les choses reprennent leur place, mais l'incertitude de l'avenir face à ce virus reste toujours. Ceci a comme impact que nous maintiendrons une partie de notre formation en ligne. Ainsi, nous aurons la possibilité de poursuivre nos activités de formation même si une deuxième vague nous frappe »(CP2).

Enfin, la pandémie met en lumière des écarts entre les CFP et entre les programmes d'un même centre, entre des CFP de différentes régions et de nombreuses stratégies collaboratives pour réduire ces écarts. Puisque les environnements variés, les modes d'enseignement diversifiés et les modalités multiples d'organisation de formation sont le propre de la FP et permettent une adaptation au contexte local et une certaine compétitivité entre les CFP, est-ce qu'une plus grande uniformité est souhaitable? Est-ce que d'autres équipes ou même d'autres secteurs de l'école publique pourraient s'inspirer de ce qui existe et de ce qui a été accompli en FP en contexte de pandémie ? Est-ce que les changements de pratiques enseignantes, changements obligés, mais réfléchis et déclarés à propos des objets de formation, des processus et des éléments organisationnels transformeront à long terme les routines, les fonctionnements, les conceptions de l'enseignement en FP ? 


\section{Notes}

1 Administration, commerce et informatique; Agriculture et pêches; Alimentation et tourisme; Arts; Bois et matériaux connexes; Chimie-biologie; Bâtiment et travaux publics; Environnement et aménagement du territoire; Électrotechnique; Entretien d'équipement motorisé; Fabrication mécanique; Foresterie et papier; Communications et documentation; Mécanique d’entretien; Mines et travaux de chantier; Métallurgie; Transport; Cuir, textile et habillement; Santé; Services sociaux, éducatifs et juridiques; Soins esthétiques.

2 «Formule éducative permettant à un apprenant de développer des compétences d'une façon autonome, accompagné par un enseignant, instrumenté de guides d'apprentissages, de matériel pédagogique et didactique.凶»(TREAQ-FP, 2017)

3 Le cas-récit en santé a été rédigé conjointement par un conseiller pédagogique (CP2), un responsable de la formation en entreprise (P1), un enseignant (E2) et une direction (D1).

4 Alors que le DEP mène l'élève « à offrir de l'aide et des soins d'assistance à une clientèle de tout âge présentant des maladies ou des incapacités physiques, psychiques ou psychosociales dans les réseaux public, privé et communautaire » (Inforoute, 2020).

5 En temps normal, le programme MICE est offert en enseignement individualisé, comporte des périodes d'enseignement collectif et $20 \%$ de la formation s'effectue en ATE, en plus d'être fortement axée sur une approche par projets.

\section{Références}

Alexandre, M. (2019). Planifier en formation à distance à l'université: reconfiguration temporelle et usage didactique des outils numériques. Dans F. Lafleur et G. Samson (dir.). Pratiques et innovations à l'ère du numérique en formation à distance: technologie, pédagogie et formation (pp. 103-116). PUQ.

Alexandre, M. (2018). Le modèle de la sémiose et le savoir-enseigner en formation à distance à l'université : Donner sens à l'explicitation. Expliciter. GREX, 118(2), 40-51.

Alexandre, M. (2017). L'apport des construits au champ didactique : le savoir-enseigner au collégial, La Revue des sciences de l'éducation de McGill. 52(3), 571-596

Altet, M. (2002). Une démarche de recherche sur la pratique enseignante : l'analyse plurielle, Revue française de pédagogie, 138(janvier-février-mars), 85-93. https:/www.persee.fr/doc/rfp 0556-7807 2002 num 13812866

Balleux, A. (2011). Lentrée en enseignement professionnel au Québec : un long parcours de transition en tension entre le métier exercé et le métier enseigné. Recherches en éducation, 11(1), 55-66. http://www.cnipe.ca/IMG/pdf/pdf Carrierologie Balleux10 3 4 603 627-2.pdf

Banque de données statistiques officielles du Québec (2018). Personnel des commissions scolaires, selon le statut et la catégorie d'emploi, le secteur et l'ordre d'enseignement et le sexe, Québec, de 2010-2011 à 2015-2016. https://bdso.gouv.qc.cal

Banque de données statistiques officielles du Québec (2019). Effectif scolaire de la formation professionnelle, selon diverses variables, années scolaires 2005-2006 à 2017-2018. https://bdso.gouv.qc.ca/

Beaucher, C., Coulombe, S., Murphy, C., Gagnon, C., Doucet, M., et D. Maltais, (2020). Les élèves à besoins particuliers en formation professionnelle: qu'en savons-nous? Dans Mazalon, É. et Dumont, M. (dir.) Soutien à la persévérance et à la réussite des jeunes et des adultes en formation professionnelle, (pp. 17-36). PUQ.

Basque, J. et Baillargeon, M. (2013) La conception de cours à distance. Le Tableau, 2(1). http://pedagogie.uquebec.ca/le-tableau/la-conception-de-cours-distance

Breton, S. (2020). La pédagogie inclusive en réponse à la diversité des besoins d'apprentissages des élèves inscrits en formation professionnelle. Dans Mazalon, É. et Dumont, M. (dir.) Soutien à la persévérance et à la réussite des jeunes et des adultes en formation professionnelle, (pp. 259-278). PUQ.

Bru, M. (1992). Les variations didactiques dans l'organisation des conditions d'apprentissage. Éditions Universitaires du Sud. 
Collin, S. et Karsenti, T. (2013). Usages des technologies en éducation : analyse des enjeux socioculturels. Éducation et francophonie, 41(1), 192-210. https://www.erudit.org/fr/revues/ef/2013-v41-n1-ef0525/1015065ar.pdf

Coulombe, S., Doucet, M., Maltais, D., DeChamplain, A. et D. Bizot. (2019). Les pratiques d'enseignement, des savoirs issus de l'expérience. Dans Doucet, M. et M. Thériault (2019) (dir.) L'adulte en formation...pour devenir soi. Espaces, passages, débats et défis (pp.171-198). Québec : PUQ.

Couture, C., Dionne, L., Savoie-Zajc, L. et Aurousseau, E. (2012). Ajustements de pratiques d'enseignants de l'élémentaire en sciences et technologie. Formation et profession, 20(3), 1-13. http://dx.doi.org/10.18162/fp.2012.140

Desgagné, S. (2005). Récits exemplaires de pratique enseignante : analyse typologique. PUQ

Dionne, L. (2007). Apprendre à lire, à écrire et à parler de sciences : un modèle pour guider la croissance d'une communauté d'apprentissage didactique. Éducation francophone en milieu minoritaire, 2(1). https://ustboniface.ca/reefmm/file/v2n1dionne.pdf.pdf

Faucher, J. et Roy, N. (2016). Le contexte et l'environnement. Dans Gagnon, C. et Beaucher, C. (dir.). Enseigner en formation professionnelle. Pour une meilleure planification et des cours plus efficaces (pp. 71-93). Chenelière.

Gagnon, C. (2020). Accompagnement les élèves dans le cadre des stages : un processus en trois temps. Dans Mazalon, É. et Dumont, M. (dir.) Soutien à la persévérance et à la réussite des jeunes et des adultes en formation professionnelle (pp. 315336). PUQ.

Gagnon, C. (2007). Arrimage des pratiques éducatives d'enseignants et de formateurs en entreprises en contexte d'alternance. Études de cas en formation professionnelle agricole, Recherches qualitatives, 27(1), 141-190. https://savoirs.usherbrooke.ca/handle/11143/933

Gagnon, C. et Beaucher. C. (2016). Les élèves en formation professionnelle. Dans Auteurs. (dir.). Enseigner en formation professionnelle. Pour une meilleure planification et des cours plus efficaces (pp. 18-46). Chenelière.

Gagnon, C. et Tremblay, C. (2016a). «La compétence comme contenu à enseigner », dans Auteurs (dir.). Enseigner en formation professionnelle. Pour une meilleure planification et des cours plus efficaces (pp. 94-114). Chenelière.

Gagnon, C. et Tremblay, C. (2016b). La macroplanification sur la compétence. Dans Auteurs. (dir.). Enseigner en formation professionnelle. Pour une meilleure planification et des cours plus efficaces (pp. 152-185). Chenelière.

Espinosa, G. (2020). La relation enseignant-élève et l'affectivité : place et importance chez le jeune adulte en contexte de formation. Dans Mazalon, É. et Dumont, M. (dir.) Soutien à la persévérance et à la réussite des jeunes et des adultes en formation professionnelle, (pp. 155-177). PUQ.

Gosselin, M., Viau-Guay, A. et Bourassa, B. (2014). « Le développement professionnel dans une perspective constructiviste ou socioconstructiviste : une compréhension conceptuelle pour des implications pratiques ", Perspectives interdisciplinaires sur le travail et la santé, 16(3). http://journals.openedition.org/pistes/4009

Guertin, D. (2012). Une démarche de pratique réflexive pour la construction du savoir professionnel. Université de Sherbrooke. https://www.usherbrooke.ca/pedagogie/fileadmin/sites/pedagogie/MPES-DPES/Donald Guertin.pdf

Le Boterf, G. (2013). Construire les compétences individuelles et collectives. Eyrolles.

Lefeuvre, G., Garcia, A. et Namolovan, L. (2009). « Les indicateurs de développement professionnel », Questions Vives, 5(11). http://journals.openedition.org/questionsvives/627

Lenoir, Y. (2012). Pour une pratique réflexive dans l'enseignement primaire. Nous avons des idées arrêtées dès que nous cessons de réfléchir. Vivre le primaire. 24(4). https://aqep.org/wp-content/uploads/2012/09/VLP Vol25No4.pdf

Leroux, J.L. (2017). Évaluation à distance des connaissances et des compétences : un processus, une approche, des ressources pour bien accompagner. Communication réalisée à la Journée de perfectionnement pour les professionnels en milieu universitaire. 7 avril 2017. Université de Sherbrooke.

Marcel,J.-F. (2004). « Le système des pratiques professionnelles de l'enseignant de maternelle », dans Marcel, J.-F. (dir.), Les pratiques enseignantes hors de la classe (pp. 61-85). L'Harmattan.

Marcel, J. F. (2005). Le développement professionnel au travers de l'évolution des pratiques enseignantes. Revue des sciences de l'éducation, 31(3), 585-606. 
Masdonati, J., Fournier, G. et Pineault, M. (2015). La formation professionnelle au Québec : le regard des élèves. Lorientation scolaire et professionnelle, 44(2), http://osp.revues.org/4590.

Mayer, R. et Deslauriers, J.-P. (2000). «Quelques éléments d'analyse qualitative. L'analyse de contenu, l'analyse ancrée, l'induction analytique et le récit de vie », dans R. Mayer, F. Ouellet, M.-C. Saint-Jacques et D. Turcotte (dir.), Méthodes de recherche en intervention sociale (pp. 159-189). Gaëtan Morin.

Mazalon, É. et Bourdon, S. (2015). Choix scolaires et obstacles à la participation des jeunes adultes non diplômés inscrits en formation professionnelle, dans C. Villemagne et J. Myre-Bisaillon (dir.), Les jeunes adultes faiblement scolarisés. Parcours de formation et d'accompagnement (pp. 165-185). PUQ.

Ministre de l'Éducation et de l'Enseignement supérieur. (2019). Cadre de référence de la compétence numérique. Gouvernement du Québec. http:/www.education.gouv.qc.ca/fileadmin/site web/documents/ministere/Cadre-reference-competence-num.pdf

Ministère de l'Éducation, du Loisir et du Sport. (2005). Cadre de référence sur la planification des activités d'apprentissage et d'évaluation - Formation professionnelle. Gouvernement du Québec. https://www.inforoutefpt.org/ministere docs/AdminInfo/CadreRef/CadreReference.pdf

Paillé, P. et A. Mucchielli (2016). Analyse qualitative en sciences bumaines et sociales. $4^{\mathrm{e}}$ édition. Armand Colin.

Philion, R., Bourrassa, M., Lanaris, C. et Pautel, C. (2016). Guide de référence sur les mesures d'accommodement pouvant être offertes aux étudiants en situation de handicap en contexte universitaire. Université du Québec en Outaouais. http://uqo.ca/docs/9752

Plamondon, M. et S. Ross, (2020). L'intégration des technologies de l'information et de la communication (TIC) dans l'enseignement. Dans Mazalon, É. et Dumont, M. (dir.) Soutien à la persévérance et à la réussite des jeunes et des adultes en formation professionnelle (pp. 235-258). PUQ.

Roberge, J. (2017). Commenter autrement les travaux des étudiants. Pédagogie collégiale, 30(3). 21-27. https://aqpc.qc.ca/sites/default/files/revue/roberge-vol 30-3.pdf

Savoie-Zajc, L., Dolbec, A., Desjardins, L. et Zniber, A. (2010). La réussite scolaire d'étudiants adultes inscrits dans des programmes de formation professionnelle: enjeux, défis. Rapport de recherche. Québec, FQRSC-MELS.

Schön, D.A. (1994) Le praticien réflexif. À la recherche du savoir caché dans l'agir professionnel. Logiques Éditions.

St-Germain, M. et Mazalon, É. (2020). La mise en oeuvre d'une relation de coopération entre enseignant et élèves pour soutenir l'apprentissage en classe et en atelier. Dans Mazalon, É. et Dumont, M. (dir.) Soutien à la persévérance et à la réussite des jeunes et des adultes en formation professionnelle, (pp. 215-232). PUQ.

TRÉAQ-FP. (2017). L'enseignement individualisé dans les centres de formation professionnelle au Québec. Table des responsables de l'éducation des adultes et de la formation professionnelle des commissions scolaires du Québec. https://www.treaq.ca/wp-content/uploads/2019/05/Rapport sondage EI 7avril17.pdf

Tremblay, C. (2016). La macroplanification sur les programmes d'études. Dans Gagnon, C. et Beaucher, C. (dir.), Enseigner en formation professionnelle : Pour une meilleure planification et des cours plus efficaces (pp.116-151). Chenelière.

Uwamariya, A. et Mukamurera, J. (2005). Le concept de développement professionnel en enseignement: approches théoriques. Revue des sciences de l'éducation, 31(1), 133-155. https://www.erudit.org/fr/revues/rse/2005-v31-n1-rse994/012361ar/

Wittorski, R. (2007). Professionnalisation et développement professionnel. L'Harmattan.

Yin, R.K. (1994). Case study research. Design and methods (2e edition). Sage publications.

\section{Pour citer cet article}

Coulombe, S., Gagnon, C., Bisson, J., Gagné, A., Dupuis, S., Larouche, M., Alexandre, M., et Beaucher, C. (2020). Transformations des pratiques enseignantes en formation professionnelle au Québec avec l'arrivée de la COVID-19. Formation et profession, 28(4 hors-série), 1-13. http://dx.doi.org/10.18162/fp.2020.682 